\title{
LAS METÁFORAS \\ EN EL LENGUAJE HOSPITALARIO
}

\author{
Pilar Darriba Rodríguez
}

Supervisora Formación Continuada de Enfermería

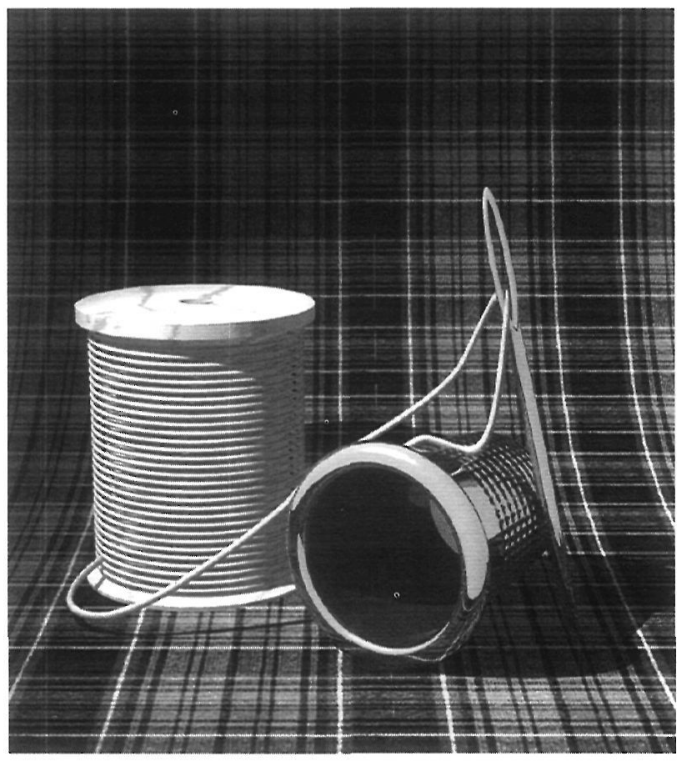

\section{RESUMEN}

Existen diferentes bases para la utilización de las metáforas, pero la experiencia física, social y cultural de cada persona proporciona los fundamentos necesarios para su utilización y elaboración. Cada "cultura" posee un lenguaje propio, lo que deriva en la utilización de unas metáforas propias de esa "cultura" en particular y en su aplicación; fuera de dicho ambiente, puede no ser comprendida e incluso interpretada de modo diferente. Las metáforas impregnan el lenguaje cotidiano hasta el punto de que muchas expresiones no podrían decirse sin utilizar metáforas. El mundo sanitario posee un lenguaje propio, que (generalmente) utiliza el personal que en él trabaja. Con este trabajo pretendo describir y analizar algunas de las metáforas utilizadas habitualmente en el ambiente hospitalario, basándome en la clasificación de Lakoff y Johnson.

Palabras clave: Enfermería, metáforas, semántica sanitaria.

\section{THE METAPHOR IN HOSPITAL LANGUAGE}

\section{SUMMARY}

There are innumerable sources from which to extract metaphors but undoubtedly physical, social and cultural experiences of human beings are probably the richest fields to turn to when needed. Every 'culture' has its own language, uses its own metaphors, some of which have no significance at all in other cultures: They could be understood in a different way, they could be misinterpreted.

Sometimes metaphors pad out every day language to such a point that many expressions could not be interpreted nowadays without those metaphors. Hospital life for instance, has its own vocabulary. This study tries to analyse and describe some of the metaphors used during day in day out hospital life using Lakoff and Johnson's classification.

Key words: Nursing, metaphor, semantics in hospital life.

Según Lakoff y Johnson, la metáfora impregna la vida cotidiana, tanto desde el lenguaje como desde el pensamiento y la acción, ya que nuestro sistema conceptual ordinario es fundamentalmente de naturaleza metafórica, aunque no seamos conscientes de ello de manera habitual.
La esencia de la metáfora es entender y experimentar un tipo de cosa en términos de otra, al mismo tiempo, esto hace que se oculten otros aspectos del concepto, que se vuelven inconscientes con la metáfora (caso del lenguaje "hospitalario" del que seguidamente voy a comentar), al 
mismo tiempo que parte de ese concepto metafórico no se ajusta ni puede ajustarse a la realidad; esta explicación pertenece a un tipo de metáforas llamado estructurales, es decir, un concepto está estructurado metafóricamente en términos de otro.

Pero, según esta clasificación, existen otros tipos de metáfora: como las metáforas orientacionales que organizan un sistema global de conceptos en relación con otros y tienen que ver con la orientación espacial, no son arbitrarios y tienen su base en la experiencia personal, física y cultural, ya que pueden variar de una cultura a otra, así dicen Lakoff y Johnson "en realidad creemos que ninguna metáfora se puede entender, ni siquiera representar adecuadamente, independiente de su fundamento en la experiencia".

Así, los valores mas fundamentales en una cultura serán coherentes con la estructura metafórica de los conceptos fundamentales de la misma; pero si las circunstancias de esos valores cambian, puede afectar ese cambio a las metáforas asociadas con ellos.

Siguiendo con la clasificación anterior nos encontramos con las metáforas ontológicas, que sirven a efectos diversos y que están basadas en la propia experiencia con objetos físicos (especialmente nuestros propios cuerpos), proporcionando una amplia variedad de este tipo de metáforas, es decir, formas y maneras de considerar acontecimientos, emociones, actividades, etc. como entidades y/o sustancias.

Muchas de estas expresiones, no solo ontológicas sino también orientacionales, no son reconocidas como metafóricas, entre las razones para ello están el que estas metáforas sirven para una gama limitada de propósitos o que se encuentran poco elaboradas.

Estos tres tipos de conceptos metafóricos presentados corresponden a áreas de experiencia básicas que permiten comprender otras experiencias en sus términos, de ahí se extrae que al hablar de gran cantidad de hechos y procesos de nuestro entorno más inmediato se utilicen expresiones provenientes de otro campo a las que les otorgamos un significado propio de cada "cultura" particular; en la mayoría de los casos las metáforas expresan realidades abstractas en términos de otras más concretas de la experiencia humana y de su universo de acción.
El lenguaje "hospitalario", como tantos otros, puede enmarcarse dentro de este mundo metafórico, así el objetivo perseguido es, que a través de la clasificación anterior, conseguir explicar su significado, utilidad e interpretación.

Así, entre las metáforas que podrían incluirse en las de tipo estructural se encuentran:

* La Radiología es la guerra:

¡dispara!

¡escapar que disparo!

¡quieto que disparo!

¡no respire!: ¡disparo!

Todas estas expresiones se refieren al hecho de realizar una radiografía $y$, en el lenguaje hospitalario y concretamente el utilizado en el Servicio de Radiología, se concibe como el momento en el que se aprieta el botón del aparato de rayos $\mathrm{X}$ y se emite la radiación necesaria para realizar la radiografía, entendiendo el: "disparo" como: "emito rayos $X^{\prime \prime}$, acción que va acompañada de la necesidad de alejarse de las radiaciones ionizantes por parte del personal que se encuentre próximo y también la necesidad por parte del paciente de no moverse con el fin de que la radiografía salga correcta. Pero entiendo que para el paciente o personas profanas al mundo sanitario y concretamente a Radiología, este tipo de expresiones puede tener un significado completamente diferente y puede llegar a preguntarse: ?dónde estoy, en un hospital o en la guerra? con lo que ello conlleva de ansiedad si previamente no se le ha explicado en que consiste la prueba.

* Los bebés son alimentos:

igambita!

¡bollito!

¡bombón!

¡cosita!

¡está para comérselo!

Los bebés son personajes tan pequeños e indefensos que cariñosamente son llamados de muy diferentes formas, entre ellas las que aparecen aquí, que curiosamente semejan que los bebés son alimentos y se les puede comer, algo que se podría explicar asimilando el placer de comer al placer que produce ver a los bebés como personas en miniatura, se asocia a algo placentero y bonito.

* La acumulación de pacientes como objeto: 
acaba de llegar el autobús

el pasillo está a reventar

Estas expresiones se refieren a la acumulación de pacientes en el servicio de Urgencias y analizándolas podemos encontrar que un pasillo no puede reventar y que un autobús no es lógico que penetre en un Servicio de Urgencias, pero si se podría ver que como la mayoría de los pacientes que acuden a un servicio de Urgencias no presentan tal urgencia y además la afluencia al Servicio está, en parte, determinada por los partidos de fútbol, el buen o mal tiempo, los lunes y cualquier otro tipo de acontecimientos, el personal sanitario asocia una gran afluencia de personas, sin una urgencia vital, a dicho Servicio con la llegada de un autobús lleno de gente y con un caos o un gran reventón o con cualquier otro acontecimiento en el que la gran afluencia de gente sea el tema central.

\section{* Los animales tienen atribuciones humanas:}

creer en los pajaritos preñados

estar con la mosca tras la oreja

tener mariposas en el estómago

Las dos primeras metáforas significan, no solo en el mundo sanitario, desconfianza y la tercera ansiedad, pero es posible ver en ellas el significado de la relación de los animales con los humanos a través de ciertas expresiones que son utópicas (en su inmensa mayoría).

* Lo que pasa en un hospital pasa en una cocina:

parece una carnicería (referido a un accidente importante)

¡vaya escabechina! (idem anterior)

¡vaya cacharrazo! (idem anterior)

jestá forrado de pasta (referido a una persona que realiza muchas horas extras)

¡tráeme el cacharro! (referido a un aparato sanitario)

tiene los ojos como platos (cuando un paciente tiene los ojos demasiado abiertos)

los pies le huelen a queso (referido al mal olor)

se va a quedar como vegetal (referido al estado vegetativo en el que se encuentra un paciente)

¡cuánto come este cáncer! (referido al adelgazamiento que produce el cáncer)
Dentro de este apartado se podrían encontrar metáforas estructurales y también ontológicas pero están agrupadas en la frase central para significar que el lenguaje utilizado en ciertos ambientes puede significar algo totalmente diferente colocado en otros ambientes diferentes, así el lenguaje culinario es aplicacble al mundo hospitalario pero su significado cambia radicalmente como se puede ver.

Dentro de las metáforas ontológicas se podrían incluir las siguientes:

* La muerte es un viaje:

no llega a Navidades

éste sale con los pies por delante

éste no la cuenta

está con un pie aquí y otro allá

pasar el "muerto" a otro

El tema de la muerte es un paso importante y trascendente en una persona y en el mundo sanitario es algo que ocurre a diario, por lo cual el personal sanitario vive frecuentemente esta situación, para evitar que influya en el estado anímico del personal sanitario que trabaja en el Hospital (algo difícil de evitar), éste se inventa metáforas que le ayuden a superar dicho trance, trivializando situaciones emocionales importantes mediante dicho lenguaje; es necesario resaltar que este tipo de lenguaje solo se utiliza entre los propios profesionales y no suele llegar al paciente, a los familiares o al público en general.

* El Hospital tiene entidad:

la casa grande

soy de la casa

la casa de locos

El Hospital, al ser un edificio grande en el que trabajan muchas personas, puede tener entidad propia, ya que en él se pasan muchas horas trabajando y es una parte importante de la vida de los trabajadores, de ahí su propia entidad.

* La tranquilidad no es tranquilidad: ¡tranquilo!

¡tranquilo, esto pasa pronto! (la enfermedad, la muerte, ...)

¡tranquilo!: iquieto!

¡tranquilo! ahora acabamos

etc. 
La palabra tranquilidad es utilizada como "coletilla" en innumerables ocasiones dentro del mundo sanitario, podría explicarse, también, de diferentes formas: en algunas ocasiones podría significar: "no me molestes", en otros podría significar una trivialización o incluso mentira acerca de su enfermedad o de su posible muerte, intentando que el paciente permanezca cómodo pero, al mismo tiempo, supone una contradicción porque el paciente conoce su estado y si lo está pasando mal, en general, lo que desea es que le proporcionen cuidados para encontrarse mejor, por lo que en ciertos casos la palabra "tranquilo" es, incluso, contraproducente. En otras ocasiones, a la palabra "tranquilo", que podría pensar que expresa un deseo de que el paciente reduzca su ansiedad, le sigue una orden, como ?quieto!, que solamente por ser una orden ya supone una alteración de su supuesta tranquilidad; y si se continuara analizando el significado de dicha palabra,sola o en el contexto de una frase, se continuaría añadiendo más significados del que realmente parecería que podría tener.

* El estado anímico es una máquina:

le falta un tornillo

le falta una tuerca

hay que darle una vuelta de tuerca

está pasado de vueltas

tiene los tornillos flojos

tiene las ideas oxidadas

le patinan las neuronas

* El estado anímico tiene música:

¡está tocado!

¡está tururú!

¡está como una jota!

¡está p'allá!

Todas estas metáforas significan el estado de la mente humana desde un punto de vista de salud mental, explicándolo en función de una máquina que no funciona o desde el punto de vista musical, expresando así la importancia que tiene dicho estado para el funcionamiento integral del resto del organismo, ya que en una máquina es fundamental para su funcionamiento el que todas sus piezas funcionen correctamente al igual que pasa en la música, dónde la coordinación entre los diferentes instrumentos musicales es importante para que el resultado final sea el esperado.
* La Radiología es una cocina:

quema un poco esa placa (radiografía)

esta placa está churruscada

¡vaya churro de placa!

¡dale kilos! (referido a kilovoltaje)

Todas estas metáforas forman parte del lenguaje cotidiano de un Servicio de Radiología, y volviendo al tema comentado anteriormente referido al Hospital como cocina, en Radiología ocurre lo mismo, al utilizar un lenguaje culinario en un ámbito diferente.

\section{* La enfermedad como objeto:}

tiene una caverna (referido a tuberculosis

pulmonar)

está invadido hasta el carnet de identidad

está infectado hasta la suela del zapato

En el lenguaje médico se utilizan con relativa frecuencia palabras médicas que tienen otros significados ademăs del propio significado científico, tal es el caso de "caverna" que utilizado en el ámbito sanitario significa una importante alteración pulmonar debida a la tuberculosis y en otro ámbito podría ser cueva; además es frecuente la metáfora que identifica un avance importante de la enfermedad con algo (puede ser un objeto) ajeno a la naturaleza humana pero que tiene mucha relación con la misma: es el caso del carnet de identidad (algo que posee toda persona) o los zapatos (idem anterior) aunque en este caso también se podría hablar de metáfora orientacional al definir la infección hacia la suela del zapato, objeto situado en el suelo, es decir, en la zona inferior calificando así a la infección de algo peiorativo.

Como conclusión se podría decir que existen diferentes bases para la utilización de las metáforas, pero la experiencia física, social y cultural de cada persona proporciona los fundamentos necesarios para su utilización y elaboración. Cada "cultura" posee un lenguaje propio, lo que deriva en la utilización de unas metáforas propias de esa "cultura" en particular y su aplicación, fuera de dicho ambiente, puede no ser comprendida e incluso interpretada de modo diferente. Las metáforas impregnan el lenguaje cotidiano hasta el punto de que muchas expresiones no podrían decirse sin utilizar metáforas. El mundo sanitario posee un lenguaje propio, que utiliza el personal que en él trabaja y en algunas ocasiones trasciende al público 
en general, y en estos casos su interpretación puede presentar ciertos problemas, por lo que sería muy importante, para el personal sanitario, empatizar con el paciente, hasta el punto de comprender su incomprensión, y utilizar un lenguaje adecuado, comprensible y adaptado a cada persona en particular, aunque entre el propio personal sanitario se utilice un lenguaje diferente, propio del medio en el que se encuentra y con significado solamente para ese grupo de personas.

\section{BIBLIOGRAFÍA}

Darriba Rodríguez P. (1999) Enfermería Radiológica: una manera de ser, estar y cuidar para humanizar la tecnología (conferencia). VIII Congreso Nacional de Enfermería Radiológica, Barcelona
De los Santos Alfonso J.M. La Ciencia del arte de cuidar. Híades. Revista de Historia de la Enfermería. 3-4: 17-25

-Reflexión sobre la palabra Enfermería. Híades. Revista de Historia de la Enfermería. 3-4: 1116

Eseverri Chaverri C. (1986) El paciente como centro de atención ?utopía o realidad? Rev. Index de Enfermería. 7: 7-11

Fernández Sola C. y otros. Importancia de la información de Enfermería Radiológica al usuario. Rev. Enfermería Radiológica. 34: (5-7)

Hernández Conesa J. (1995) Historia de la Enfermería. Un análisis histórico de los cuidados de enfermería. Ed. Interamericana McGraw-Hill, Madrid

Pinos T. (1995) Hazañas Médicas. Planeta, Barcelona.

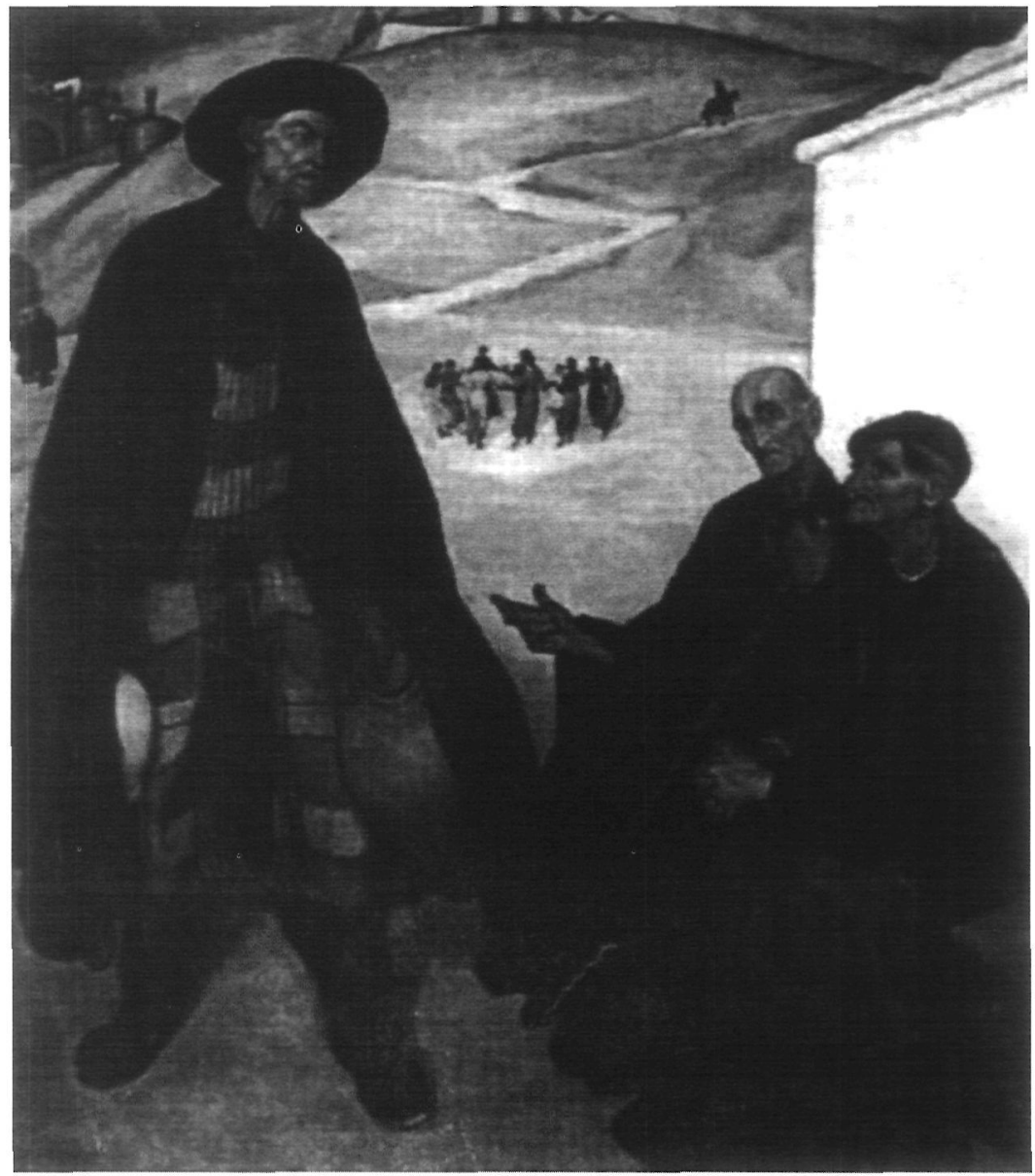

